\title{
Solid Lipid Nanoparticles: A Promising Technology for Delivery of Poorly Water-Soluble Drugs
}

Shailendra Bhatt ${ }^{1 *}$, Jaibharti Sharma ${ }^{1}$, Manishkumar Singh ${ }^{1}$, Vipin Saini²

\author{
1 MM College of Pharmacy, Pharmaceutics, Ambala, India.
}

2 MM University, Solan, India.

\section{ABSTRACT}

Oral administration of drugs having low water solubility is hampered by various enzymatic barriers present in gastrointestinal (GI) tract. Lipid nanoparticles based on solid matrix have emerged as a potential drug delivery system to improve the absorption and bioavailability of several drugs, especially lipophilic compounds. Solid lipid nanoparticles (SLN) are reported as the most promising technology for oral administration and offered several advantages over conventional dosages formulations including, enhancement in solubility, stability, permeability, and bioavailability with minimal side effects. In this review, we have highlighted recent advances in the development of SLN for the oral, parenteral, rectal, and topical administration of various drugs. We have also summarized the applications of SLN in the treatment of various diseases like tuberculosis, cancer, diabetes, and several nervous system related disorders.

Keywords: Bioavailability, SLN, permeability, target delivery

\section{INTRODUCTION}

The most demanding research area in pharmaceutical field is target delivery of a drug to a specific organ. Whenever a drug is formulated, the dosage form is chosen according to its physicochemical and biological properties. It is inconvenient to take some drugs in simple dosage form because of their low aqueous solubility and low permeability across the cell membrane. Controlled drug delivery system, either dissolution-controlled or diffusion-controlled is used for the delivery of such drugs $^{1}$. For the controlled delivery of a drug, colloidal systems such as liposomes, micelles and nanoparticles have been developed. Recently, nanotechnology has made some considerable advances in controlled drug delivery systems including 
sustained release, timed release, extended release, and targeted release of a drug. The predominance of nanoparticles over the other drug delivery systems enables them to use for drug targeting. Nanoparticles are 10-10onm in size in which drug is either dispersed or entrapped in a matrix, encapsulated as the solid solution or may be adsorbed on the surface ${ }^{2}$.The nanoparticles are designed to provide intimate contact with GI epithelium, prolong residence time and allow permeation across the cell membrane. A pre-systemic metabolism of a drug can be avoided by using nanoparticles ${ }^{3}$. Lipid nanoparticles are safe to use as they contain lipids, which are included in the category of generally recognized as safe (GRAS).

\section{Solid Lipid Nanoparticles}

Solid lipid nanoparticles are an advanced and rapidly budding field of nanotechnology with several applications in pharmaceutical science. SLN were developed first in 1991. These are the colloidal carriers possesses an average diameter of 10 to 1000nm. They composed of single lipid core matrix dispersed in an aqueous surfactant solution. The carriers like liposomes, polymeric micelles, and porous solids can also be used to enhance the aqueous solubility of a drug. But SLN not only helps to increase the solubility of a drug but also offers target release of drugs. SLN can be used as a carrier for lipophilic as well as hydrophilic drugs ${ }^{4}$. SLN provides stability to the solid lipid matrix and overcomes the disadvantages of liposomes, which includes stability problems ${ }^{5}$. Instead of polymeric nanoparticles, SLN are found to be more compatible with the biological system. Exclusive properties of SLN such as smaller size, larger surface area and interaction of phases at the interface make them valuable candidate ${ }^{2}$. The release of encapsulated compounds is controlled by solid lipid matrix, which also improves the stability of incorporated lipophilic ingredients. The type of lipids, surfactants and their concentration in the formulation of SLN adjust the particle size, drug loading, and release behavior of drugs ${ }^{5}$. The physicochemical characteristics of the solid lipid phase, such as reduced mobility of the drug in solid matrix, micro phase separation and enhanced absorption of poorly water-soluble drugs offer potentially beneficial effects. The release of a lipophilic drug from SLN was found to be better than compare to the emulsion. SLN also improves the solubility and bioavailability of lipophilic drugs ${ }^{6}$. SLN possess controlled drug release through lipid matrix and protect the drug from gastric degradation?. They improve the stability of those drugs which are sensitive to light, environment, $\mathrm{pH}$, and oxidation ${ }^{8}$. The incorporated drugs can be protected against enzymatic degradation ${ }^{6}$. SLN enhance the intracellular uptake of drugs and deliver them at defined rates ${ }^{8}$.

\section{Role of lipids and emulsifiers in the formulation of SLN}

Table 1 show some examples of lipids and surfactants, which are used in the for- mulation of SLN.

\section{Lipids}

Main features for the selection of lipid are as following:

- Lipids carriers should enhance the gastrointestinal solubility and improve lymphatic uptake of poorly bioavailable drugs ${ }^{9}$.

- The chemical and physical behavior of the $\operatorname{lipid}^{10}$.

- The lipid with long chain fatty acids should be considered as they crystallize slower than short chain fatty acid ${ }^{11}$.

- Proper care should be taken while selecting the lipid, as some lipids convert from metastable to stable form and try to form ideal crystalline lattice structures $^{11}$.

- The lipid influences the drug loading capacity in SLN. The higher amount of drug in lipid results in the more un-entrapped drug in the formulation ${ }^{12}$.

- The size of SLN is also influenced by the hydrophobic part of the lipid and found to be increased when the hydrophobic chain length of lipid increases ${ }^{13}$.

- The non-erodible and non-digestible properties of lipid ${ }^{14}$.

- The selection of lipid depends upon the solubility of the drug in the lipid. In a study, the anticancer drug wogonin showed the highest solubility in the stearic $\operatorname{acid}^{15}$.

Glyceryl behenate is an ideal excipient for SLN and has been used by various research groups. The stability and zeta potential of SLN prepared with glyceryl behenate is affected by storage conditions ${ }^{16}$. Jannin et al. reported that SLN prepared with glyceryl dibehenate were not digested by pancreatin during in-vitro lipolysis test ${ }^{14}$. In one of the study, it was found that SLN prepared with glyceryl behenate showed higher drug entrapment than compare to glyceryl stearate, as it contains mono, di and triglycerides and produces less ordered lipid crystals than glyceryl stearate ${ }^{17}$. In a study, SLN prepared using glycerol monostearate, stearic acid and glyceryl behenate resulted in an increase in particle size, polydispersity index and entrapment efficiency after long storage. However, the SLN containing tristearin as lipid content was found to be stable ${ }^{18}$. Citral loaded SLN, using glyceryl monostearate as a lipid showed enhanced stability in acidic enviroment ${ }^{19}$. Chantaburanan et al. prepared ibuprofen SLN to evaluate the combined effect of wax (cetyl palmitate) and triglyceride (Softisan 378) on particle size, zeta potential, entrapment efficiency, crystallinity, and drug release. The SLN were resulted in smaller particle size. The drug release was found to be fast followed by sus- 
tained release, depending upon the ratio of lipids used ${ }^{20}$. In a study, ketoprofen loaded SLN were prepared using beeswax and carnauba wax. SLN containing higher amount of beeswax showed faster drug release as compared to SLN with the higher amount of carnauba wax ${ }^{21}$.

Table 1. List of Lipids and Surfactants used in the formulation of SLN

\begin{tabular}{|c|c|}
\hline Lipids & Surfactants \\
\hline $\begin{array}{l}\text { Fatty acids: Behenic acid, Stearic acid, Myristic } \\
\text { acid, Palmitic acid, Decanoic acid. }\end{array}$ & $\begin{array}{c}\text { Sorbitan ethylene or propylene oxide } \\
\text { copolymers: Polysorbate } 60 \text {, Polysorbate } 20 \text {, } \\
\text { Polysorbate } 80 \text {. }\end{array}$ \\
\hline $\begin{array}{c}\text { Acylglycerols: Glycerol palmitostearate, Glyceryl } \\
\text { monostearate, Glyceryl behenate, Glyceryl } \\
\text { hydroxy stearate. }\end{array}$ & $\begin{array}{c}\text { Ethylene oxide or propylene oxide copolymers: } \\
\text { Poloxamer 407, Poloxamine 908, Poloxamer 188, } \\
\text { Poloxamer } 182 \text {. }\end{array}$ \\
\hline $\begin{array}{l}\text { Triacylglycerides: Tripalmitin, Trimyristin, } \\
\text { Tricaprin, Tristearin, Trilaurin. }\end{array}$ & Alkylaryl polyether alcohol polymers: Tyloxapol. \\
\hline $\begin{array}{l}\text { Waxes: Beeswax, Cetyl palmitate, Carnouba } \\
\text { wax. }\end{array}$ & $\begin{array}{l}\text { Triacylglycerides: Tripalmitin, Trimyristin, } \\
\text { Tricaprin, Tristearin, Trilaurin. }\end{array}$ \\
\hline Cyclic complexes: Cyclodextrin. & $\begin{array}{l}\text { Bile salts: Sodium cholate, Sodium oleate, } \\
\text { Sodium glycocholate, Sodium taurodeoxycholate, } \\
\text { Sodium taurocholate. }\end{array}$ \\
\hline Hard fat: Witepsol H 35, Witepsol W 35. & Alcohol: Butanol. \\
\hline
\end{tabular}

\section{Digestibility of Lipids}

The main problem associated with SLN is the digestibility of lipids by lipases secreted by stomach or pancreas, which further cause degradation of SLN. Lipids are considered as non-erodible and non-digestible. But the presence of surfactant allows lipases to adsorb at the interface when the interfacial tension is sufficiently lowered. Jannin et al. prepared SLN of glyceryl dibehenate to evaluate the digestibility. SLN of Glyceryl dibehenate were produced by two processes, first high-shear homogenization followed by ultra-sonication and second Spray-Flash Evaporation (SFE) method. SLN prepared by ultra-sonication showed a limited digestion of the lipid excipient when tested in-vitro lipolysis using a pH-stat apparatus, although SLN produced by SFE were not digested by lipases. So, it was concluded that the presence of surfactant allows the adsorption of the lipase and degradation of glyceryl behenate ${ }^{14}$.

\section{Surfactants}

Surfactants are used for the stability of the SLN dispersion and to prevent aggregation of particles. Various research groups have tried different surfactants like tween 20, tween 60, tween 80, span 20, polyvinyl alcohol, cremophor, lecithin, sodium dodecyl sulphate, sodium glycolate, poloxamer 407, and poloxamer 188 .
The smaller particle size can be achieved by using higher surfactant ratio. Many of the Surfactant contains the hydrophobic as well as the hydrophilic moiety. The size of SLN decreases with the shorter chain length of hydrophobic part of surfactant. While the stability of formulated SLN decreases with increase in hydrophobic chain length because of the decrease in zeta potential. The HLB value is generally taken into consideration for the selection of surfactant. Sometimes surfactant blend also used to improve stability. The particle size and entrapment efficiency depend upon the concentration of surfactant used. Sun et al. investigated that by increasing the amount of pluronic F68 in the SLN formulation, particle size decreased, and the entrapment efficiency significantly increased ${ }^{22}$. Poloxamer 407 slow down the enzymatic degradation while cholic acid and sodium salts accelerate degradation, tween 80 showed no pronouncing effect on it. Polyethylene glycol as a surface coating material slow down the clearance and prevent liver uptake as it is less prone to plasma protein and other biological materials. Polysorbates coated SLN can effectively cross blood-brain barrier than compare to poloxamer and cremophor. A study reported that the addition of TPGS (Tocopheryl Polyethylene Glycol Succinate) as an emulsifier may improve the solubility, stability and oral bioavailability of entrapped drug ${ }^{12}$.

\section{Other Agents}

Generally SLN are insufficient for the oral administration of various phyto bioactive compounds, due to burst release property ${ }^{23}$. To overcome this problem surface modifiers are used to improve the oral delivery of phyto bioactive compounds. The list of some counter-ions and surface modifiers are shown in Table $2^{11}$. Surface modification using counter-ions and surface modifiers elaborates the new physicochemical properties of SLN ${ }^{11}$. The coating of a polymer on the SLN surface improves the stability of SLN in acidic medium and prevents the burst release of drug ${ }^{24}$. The quarternized chitosan derivative can be used for the protection of drug from acidic medium and further to stabilize the SLN. The curcumin-loaded SLN coated with N-carboxymethyl chitosan (NCC) by surface charge interaction prevented the immediate release of curcumin in acidic medium and improved its bioavailability ${ }^{24}$. Ramalingam et al. prepared SLN using N-trimethylchitosan (TMC). The curcumin loaded SLN were successfully delivered to the brain via oral administration $^{12}$. Wang et al. prepared cross-linked polymer coated SLN which showed improvement in the physicochemical properties of SLN ${ }^{25}$.

\section{Preparation of solid lipid nanoparticles}

The choice of method for the preparation of SLN depends upon the type of lipid and surfactant. Table 3 shows the formulation methods along with their advantages and disadvantages. 


\section{High pressure homogenization}

In this method, the liquid is driven by homogenizers through a narrow orifice by applying high pressure. It is suitable for the production of SLN with high lipid content $^{22}$. Two approaches work on the concept of high pressure homogenization $(\mathrm{HPH})$ are; hot homogenization and cold homogenization, as shown in (Figure 1). In a study, SLN of efavirenz were prepared using this technique was found to be acceptable in term of particle size, Polydispersity index, and entrapment efficiency ${ }^{6}$.

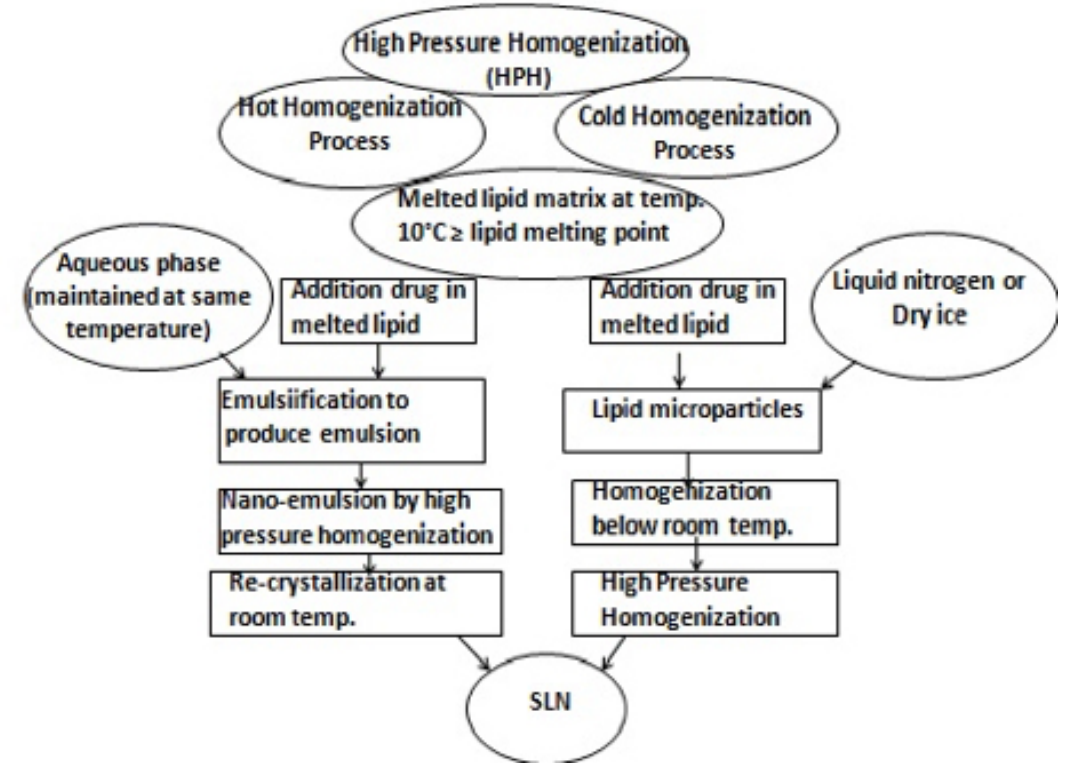

Figure 1. High Pressure Homogenization (HPH)

Table 2. List of some counter-ions and surface modifiers ${ }^{11}$

\begin{tabular}{|c|c|}
\hline Counter-ions & Surface modifiers \\
\hline $\begin{array}{c}\text { Mono-hexadecyl phosphate, } \\
\text { Sodium hexadecyl phosphate. }\end{array}$ & $\begin{array}{c}\text { a-methoxy- polyethyleneglycol 2000-carboxylic } \\
\text { acid-a-lipoamino acids, } \\
\text { a-methoxy- polyethyleneglycol 5000-carboxylic } \\
\text { acid-a-lipoamino acids. }\end{array}$ \\
\hline Dextran sulphate sodium salt. & Stearic acid-polyethyleneglycol 2000. \\
\hline Mono-decyl phosphate. & $\begin{array}{c}\text { Dipalmitoyl-phosphatidyl-ethanolamine- } \\
\text { polyethylene glycol 2000. }\end{array}$ \\
\hline Mono-octyl phosphate. & $\begin{array}{c}\text { Distearoyl-phosphatidyl-ethanolamine-N- } \\
\text { polyethyleneglycol 2000. }\end{array}$ \\
\hline
\end{tabular}

\section{Ultra-sonication/High speed homogenization}

In this method, SLN are prepared by using sonicator or high speed homogenizer. The combination of both techniques can also be used to get smaller particle size ${ }^{24}$. In a study, SLN of non-steroidal anti-inflammatory drugs were prepared using ultra-sonication assisted nano-emulsion method to get the nano-sized emulsion. The author stated that sonication for 15 minutes is necessary to obtained smaller particle size 4 . Ultra-sonication method is preferred over micro emulsion technique to get the smaller particles ${ }^{26}$.

\section{Solvent emulsification evaporation}

In this method, a water-immiscible organic solvent is used. This technique is generally used for thermo-labile drugs ${ }^{27}$.

\section{Solvent emulsification diffusion}

In this method, the lipid matrix containing drug is dissolved in a water-immiscible organic solvent and then emulsified in an aqueous phase to get SLN (Figure 2).

Lipid matrix in water immiscible organic solvent

$$
\text { emulsification in aqueous phase }
$$

$$
\checkmark
$$

Precipitation of lipid to form dispersion

\section{$\nabla$}

Evaporation of solvent in reduced pressure

SLN Dispersion

\section{Figure 2. Solvent Emulsification-Diffusion Method}

\section{Supercritical Fluid Technology}

This technique is used for the production of SLN of water-insoluble drugs. Carbon dioxide solution is the best choice as a solvent. Chattopadhyay et al. developed SLN of ketoprofen and indomethacin using this technique. The SLN with smaller particle size were obtained ${ }^{28}$.

\section{Micro Emulsion Method}

In this method, hot micro emulsion is prepared which is further dispersed in cold water with continuous stirring as shown in Figure 3. SLN using double emulsion method can also be used to overcome the problems like release of drug during 
emulsification process ${ }^{13}$. Behbahani et al. encapsulated curcumin in SLN using micro emulsion method followed by ultra-sonication. The spherical shape SLN with uniform size distribution was obtained ${ }^{5}$.
Step 1

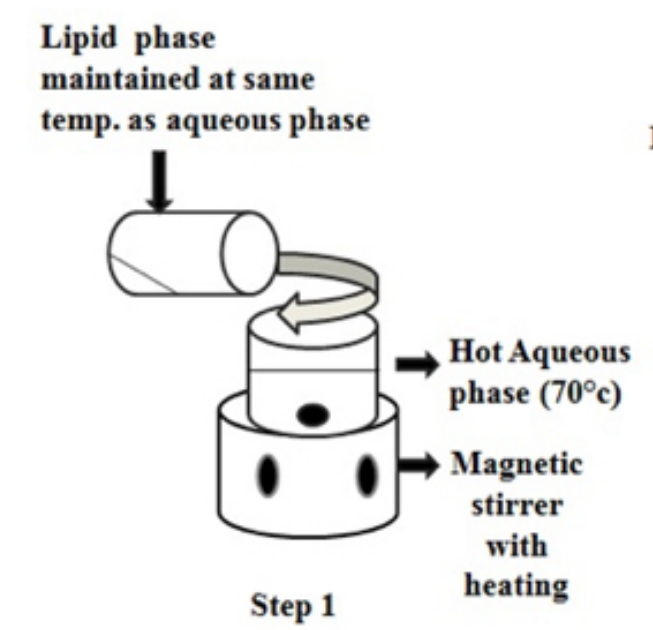

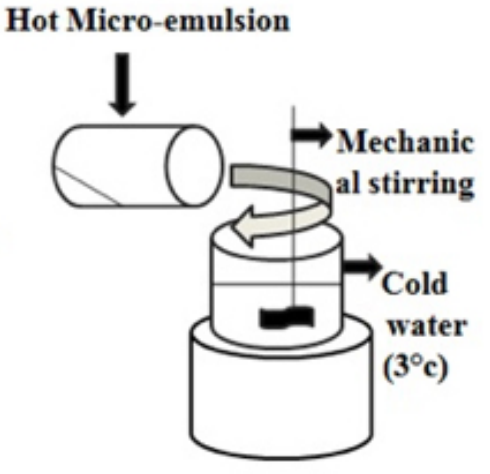

Step 2
Figure 3. Micro-emulsion method

\section{Spray Drying Method}

In this technique, high melting point lipids are used. The re-dispersion of SLN after drying is the main problem with this method. To overcome this problem, Wang et al. prepared SLN using the layer by layer technique followed by nano spray drying method to get ultra fine powders. Particles obtained were spherical in shape and easily redispersed ${ }^{29}$.

\section{Double Emulsion Based Method}

The process involves the preparation of w/o micro emulsion which is added to aqueous surfactant solution to obtain a clear w/o/w system. This double emulsion is then dispersed in cold water and further washed with dispersion medium using ultra-filtration system. Severino et al. prepared SLN of polymyxin by w/o/w emulsification method, which showed appropriate particle size (200nm), high entrapment efficiency (90\%) and desired zeta potential (-30mV). The method was found to be useful for the preparation of $\mathrm{SLN}^{30}$.

\section{Solvent Injection Technique}

In this method, the organic solvent and lipid mixture is slowly injected into aqueous surfactant solution with continuous stirring27. The resulted dispersion is then sonicated for about 12 minutes to get the SLN dispersion (Figure 4). The solvent injection-lyophilization is a modification of solvent injection method, which overcomes the problems of solvent injection ${ }^{31}$.

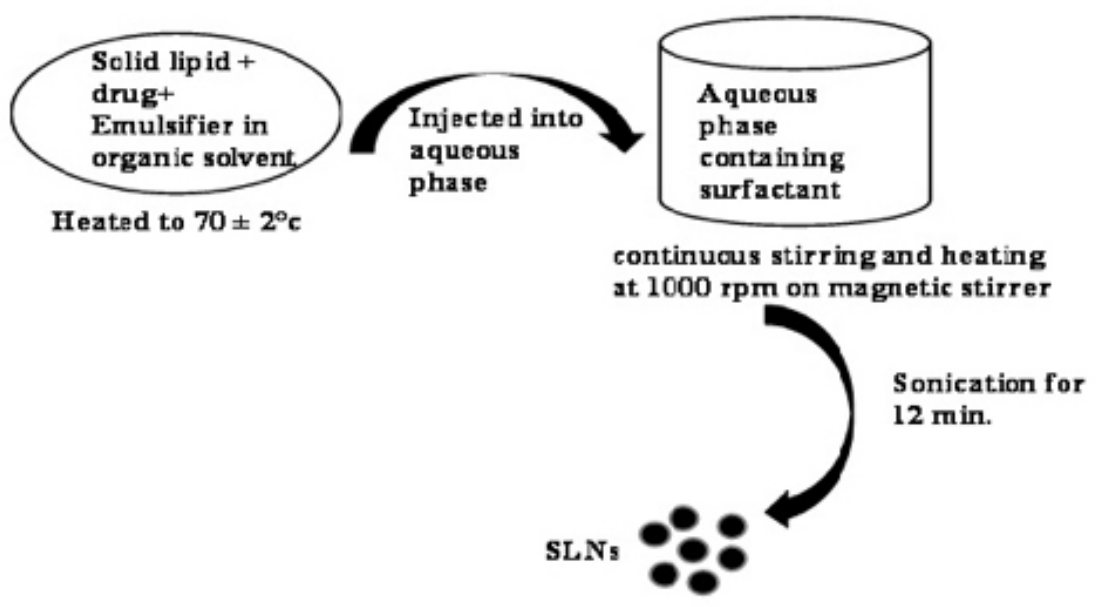

Figure 4. Solvent Injection Method.

\section{Membrane Contractor Method}

This process is developed by Charcosset et al. ${ }^{32}$. In this method, the lipid phase is pressed through the membrane pores and allows the formation of small droplets at the pore outlets, which are swept away by the running water. The SLN obtained after cooling the preparation at room temperature.

\section{Models for Incorporation of Active Compounds}

For the incorporation of active compound in SLN, three types of models are used (Figure 5). In Type I model, drug is homogenously dispersed in lipid matrix and released through diffusion from the solid lipid matrix. Type II model is achieved when the drug concentration is low and present in the outer shell. In Type III, The concentration of the drug is high in the lipid melt; a drug enriched core is formed. The drug release is governed by the fick's law of diffusion.
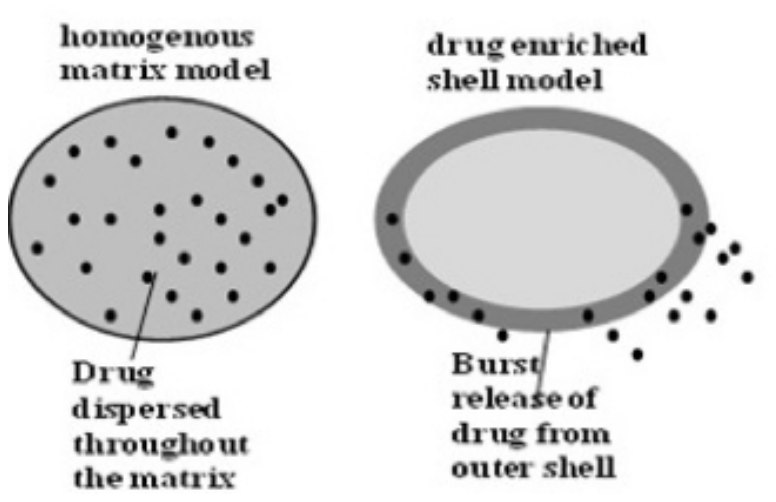

drug enriched core model

Figure 5. Drug Incorporation Models 


\section{Stability of SLN}

Any change in particle size, appearance, drug content, zeta potential, and viscosity with time can be used to determine the stability of prepared SLN. For long-term stability, parameters like temperature and light are to be observed. The most acceptable temperature condition for storage of SLN is $4^{\circ} \mathrm{C}$. For long term storage $20^{\circ} \mathrm{C}$ is preferred because at this temperature SLN are stable. While on increasing the temperature up to $50^{\circ} \mathrm{C}$, rapid growth in particle size is observed. Gaber et al. developed gelucire-based solid lipid nanoparticles of myricetin for protection of drug from degradation. The author stated that antioxidant should be added in the SLN for the protection at high temperature and buffers should be added along with surfactants for better protection of drug ${ }^{33}$.

Table 3. Method of preparation along with their advantages and disadvantages

\begin{tabular}{|c|c|c|c|}
\hline Sr. No. & Formulation procedure & Advantages & Disadvantages \\
\hline 1. & $\begin{array}{l}\text { High pressure } \\
\text { homogenization }\end{array}$ & $\begin{array}{c}\text { Economical, possible at } \\
\text { laboratory scale }\end{array}$ & $\begin{array}{l}\text { Polydisperse distribution, energy } \\
\text { intensive process }\end{array}$ \\
\hline 2. & Ultra-sonication & $\begin{array}{l}\text { Reduced shear stress, } \\
\text { avoid temperature } \\
\text { induced degradation }\end{array}$ & $\begin{array}{l}\text { Possibility of metal contamination, } \\
\text { particle growth upon storage }\end{array}$ \\
\hline 3. & $\begin{array}{l}\text { Solvent emulsification } \\
\text { evaporation }\end{array}$ & $\begin{array}{l}\text { Easily scalable, } \\
\text { continuous process }\end{array}$ & $\begin{array}{l}\text { Polydisperse distributions, energy } \\
\text { intensive process, biomolecule } \\
\text { damage }\end{array}$ \\
\hline 4. & $\begin{array}{c}\text { Solvent emulsification } \\
\text { diffusion }\end{array}$ & $\begin{array}{l}\text { Avoid heat during } \\
\text { preparation }\end{array}$ & $\begin{array}{l}\text { Residual organic solvent, anergy } \\
\text { intensive process }\end{array}$ \\
\hline 5. & $\begin{array}{l}\text { Supercritical fluid } \\
\text { technology }\end{array}$ & $\begin{array}{l}\text { Particles are obtained } \\
\text { in form of dry powder, } \\
\text { low pressure and } \\
\text { temperature conditions }\end{array}$ & High cost \\
\hline 6. & Micro emulsion & $\begin{array}{l}\text { Low mechanical energy } \\
\text { input, theoretical } \\
\text { stability }\end{array}$ & $\begin{array}{l}\text { Extremely sensitive to changes, } \\
\text { low yield }\end{array}$ \\
\hline 7. & Solvent injection & $\begin{array}{l}\text { Fast production } \\
\text { of SLN, process } \\
\text { without technically } \\
\text { sophisticated } \\
\text { equipment }\end{array}$ & Use of organic solvent \\
\hline 8. & Double emulsion & $\begin{array}{c}\text { Sterically stabilized, } \\
\text { Suitable for hydrophilic } \\
\text { drugs. }\end{array}$ & $\begin{array}{l}\text { High percentage of particles in } \\
\text { micro range. }\end{array}$ \\
\hline
\end{tabular}

\section{Characterization of SLN}

Several parameters like particle size, entrapment efficiency, zeta potential, drug loading capacity, and degree of crystallinity affect the stability and release of drug from SLN. The parameter along with their characterization techniques are given in Table 4.

The particle size of the lipid nanoparticles is strongly affected by the concentration of surfactants and lipids. Generally, the smaller particle size is obtained with the high concentration of surfactant17. Lipid concentration above $5 \%$ results in larger particle size. Zeta potential below $-25 \mathrm{mV}$ and above $+25 \mathrm{mV}$ are required for full electrostatic stabilization of the formulation. The main factor which may not show an exact result in characterization is entrapment efficiency ${ }^{34}$. Entrapment efficiency may affect by the concentration of lipid and surfactant. The loading capacity of the drug in the lipid depends upon the solubility of the drug in the lipid, structure of lipid matrix, and physical state of lipid. The solvent removal process brings some changes in lipids which can be determined using differential scanning calorimetry (DSC) and X-ray scattering. The nuclear magnetic resonance (NMR) and electron spin resonance (ESR) techniques are used for the investigation of structures in nano compartments of the lipid dispersions. The structure of lipids can also be determined using spectroscopic methods like infra-red and raman spectroscopy. Any modification in functional groups can be detected by NMR spectroscopy.

\section{Routes of Administration for SLN}

SLN can be designed for all the existing routes enlisted below.

\section{Oral administration}

SLN improves absorption of the drug through GI tract and increases oral bioavailability of drugs when used as a carrier in oral route. In one of the study, famotidine loaded SLN showed prolonged release and 2.06-fold increase in oral bioavailability as compared to commercial product7. Aripiprazole is an antipsychotic drug, the oral administration showed a reduction in in-vivo effects due to rapid first-pass metabolism. SLN of aripiprazole was developed by Sinha et al. using tristearin as a lipid and sodium taurocholate, tween 80 as surfactants. The in-vivo efficacy was found to be increased in lace mice using dizocilpine-induced schizophrenic model. The pharmacokinetic studies of SLN formulation showed 1.6 fold higher oral bioavailability of aripiprazole compared to its suspension ${ }^{35}$. 
Table 4. Parameters for Characterization of Solid lipid nanoparticles

\begin{tabular}{|c|c|c|}
\hline Sr. No. & Parameters & Characterization Methods \\
\hline 1. & Particle size measurement & $\begin{array}{l}\text { Scanning electron microscopy, photo correlation } \\
\text { spectroscopy, transmission electron microscopy, } \\
\text { atomic force microscopy, size exclusion } \\
\text { chromatography, laser defractrometer }\end{array}$ \\
\hline 2. & Charge determination & Zeta potentiometer, laser dopler anemometer \\
\hline 3. & $\begin{array}{l}\text { Loading capacity and entrapment } \\
\text { efficiency }\end{array}$ & $\begin{array}{l}\text { Spectro fluorophotometry, high-performance liquid } \\
\text { chromatography, UV-spectrophotometry }\end{array}$ \\
\hline 4. & Release profile & In-vitro release studies \\
\hline 5. & $\begin{array}{l}\text { Crystallinity and modification of } \\
\text { lipid }\end{array}$ & DSC, X-ray diffraction \\
\hline 6. & Drug excipient Interaction study & DSC \\
\hline 7. & Drug stability & $\begin{array}{l}\text { Bioassay of drug extract from nanoparticles, } \\
\text { physical and chemical analysis of drug }\end{array}$ \\
\hline 8. & Co-existence of extra structures & $\begin{array}{l}\text { Nuclear magnetic resonance, electron spin } \\
\text { resonance }\end{array}$ \\
\hline
\end{tabular}

\section{Parenteral administration}

The SLN due to their small size can travel through the vascular system and minimize the side effects of the administered drug. SLN contain physiologically welltolerated lipids which are safe to use for parental administration. They prevent macrophage uptake in the systemic circulation. Targeted delivery of hyaluronic acid coated SLN were reported by Zhou et al. In this study, SLN containing glucocorticoid prednisolone were targeted to affected joint tissues which resulted in reduction in joint swelling and inflammatory cytokines level in blood ${ }^{36}$.

\section{Topical administration}

Topical treatment with SLN delivers a higher amount of drug at the specific site of action and shows minimum side effects. Topical preparations may be used for surface effects, stratum corneum effect and systemic effects. Auraptene is an antiinflammatory agent; low aqueous solubility restricts the use for topical administration. The SLN of auraptene for topical delivery were developed by Daneshmand et al., which showed enhanced cutaneous uptake along with desirable anti-inflammatory activity ${ }^{37}$. SLN are the best way for the delivery of antifungal drugs for topical administration. The SLN gel of fluconazole was prepared to treat pityriasis versicolor. The prepared gel showed improved therapeutic activity against pityriasis versicolor compared to candistan ${ }^{\circledR} 1 \%$ cream $^{38}$.

\section{Rectal administration}

This route is preferred for pediatric patients for the ease of application. The plasma level and therapeutic effectiveness of rectally administered drugs were reported to be high as compared to oral or intramuscular route in the similar doses. Din et al. developed SLN loaded dual-reverse thermosensitive hydrogel of flurbiprofen. In comparison with the drug and hydrogel, SLN loaded hydrogel showed higher drug release than compare to pure drug and lower drug release than compare to hydrogel; due to its slow initial burst effect ${ }^{39}$.

\section{Nasal administration}

Nasal route is preferred for the fast absorption and rapid onset of action of the drug. As it avoids the GI tract thus prevent the drug degradation. The SLN of efavirenz, when administered through intra-nasal route, drug concentration in the brain was found to be increased6.

\section{Ophthalmic administration}

The main problem with the ophthalmic administration of a drug is poor bioavailability. Only $5 \%$ of the drug can pass through the cornea after administration 40. In a study, ophthalmic administration of voriconazole SLN showed burst release followed by sustained release. The prolonged residence time of drug on the ocular surface was observed ${ }^{26}$.

\section{Pulmonary administration}

SLN dispersions in the form of aerosols can be delivered to lungs, which evade the first-pass metabolism of the drug and further increase the drug absorption. The drug showed controlled release behavior when entrapped in SLN. In a comparative study of SLN and liposomes, after pulmonary administration, SLN showed prolong exposure to lung ${ }^{41}$. This may be useful for the delivery of some drugs. The paclitaxel SLN were formulated by Rosiere et al. for the lung tumor. The SLN were coated with chitosan and a folate-grafted copolymer of polyethylene glycol and delivered by pulmonary route. The coated SLN resulted in good pharmacokinetic profile, prolonged release of paclitaxel in lungs, and found to be effective in inhaled therapy of paclitaxel ${ }^{42}$.

\section{Recent Work on Solid Lipid Nanoparticles}

\section{SLN for genetic materials}

SLN have the potential for the delivery of DNA, miRNA, siRNA, nucleic acids and other genetic materials. Special cationic SLN are used for the delivery of genetic materials. Botto et al. prepared cationic SLN. The thermal analysis of cationic SLN showed the interaction with plasmid DNA. The result indicated that the efficient 
delivery of gene therapy depends upon the amount and type of surfactant used in the preparation ${ }^{43}$. Kotmakci et al. developed cationic SLN for delivery of RNAimediating plasmid DNA to reduce the over expression of STAT3 and to overcome the resistance of cisplatin in lung cancer cells. Cationic SLN of plasmid DNA complexes down-regulated the STAT3 expression in resistant cell lines and decreases the resistance of cisplatin in cells ${ }^{44}$. Sharma et al. developed TRPV1-targeting siRNA loaded SLN without using any cationic agent. The author demonstrated that PEG60o as a surfactant decreases the particle size and also affects the endosomal uptake. After topical administration of TRPV1-targeting siRNA loaded SLN to rat, it was proved that SLN are effective for the silencing of the skin TRPV1 ${ }^{45}$. Ekbaba et al. developed SLN of shRNA-encoding plasmid to reduce the level of 5- $\alpha$ reductase enzyme; which is responsible for diseases like benign prostatic hyperplasia, prostate cancer, and androgenic alopecia. The formulation was found to be efficient to reduce the level of 5- $\alpha$ reductase enzyme in the DU-145 cell line in-vitro with minimum cytotoxicity ${ }^{46}$.

\section{SLN in tuberculosis disease}

In a study, Costa et al. prepared SLN of isoniazid by functionalized with mannose (M-SLN). The M-SLN showed more uptakes on alveolar macrophage cell than the SLN. The M-SLN was found to be more effective for the delivery of anti tubercular drug to alveolar macrophages ${ }^{47}$. In another study, SLN surface were engineered with mannose for the delivery of rifampicin, for treatment of tuberculosis. The Mannosylated SLN found feasible to maintain the drug load before entering the macrophage and cause phagocytosis of alveolar macrophage ${ }^{48}$. Vieira et al. prepared chitosan coated rifampicin SLN. The chitosan coated SLN showed highest mucoadhesive properties in-vitro and higher drug release in alveolar epithelial cells A549 than compare to uncoated SLN ${ }^{49}$.

\section{SLN in cancer}

SLN have numerous applications in various types of cancer. In a study, solid lipid nanoparticles of curcumin and piperine using TPGS (tocopheryl polyethylene glycol succinate) and Brij 78 as a co-delivery vehicle was prepared. SLN were found to be effective in, in-vitro studies of reversing P-glycoprotein-mediated multidrug resistance in A2780/Taxol cells. Curcumin SLN selectively killed tumor cells with low intrinsic toxicity ${ }^{5}$. The bioavailability of solid lipid nanoparticles can be increased by surface modification with N-carboxymethyl chitosan(NCC). The intestinal delivery, lymphatic uptake, and cytotoxicity were enhanced by surface modification of SLN with NCC. The bioavailability and effect on tumor cells was found to be increased, as the cellular uptake on MCF-7 cells was increased ${ }^{24}$. A study on HCT-116 colon cells by Chirio et al. proved that the SLN can incorporate the higher amount of drug and can be used for the treatment of cell cultures with low cytotoxicity ${ }^{51}$. Chuang et al. developed pH-sensitive cationic polyoxyethylene SLN of camptothecin (CPTPEG-SLNs+) to make it tumor targeting. CPT-PEG-SLNs+ showed accumulation for a long time ( $>120$ hours) in several types of tumor, and increase the efficiency of camptothecin against $\mathrm{HCC}_{3} 6$ or CL1- 5 tumors ${ }^{52}$. Badawi et al. prepared SLN of pomegranate extract to increase its anticancer activity. The study was conducted using the normal cell line and several cancer cell lines. It was found that the SLN of pomegranate extract were more selective for the cancer lines especially for MCF-7 breast cancer cell than the normal cell ${ }^{53}$. The SLN of doxorubicin in combination with sclareol prepared by Oliveira et al. found to be useful in the treatment of cancer as they showed activity against $4 \mathrm{~T} 1$ cells with prolonged drug release ${ }^{54}$.

\section{SLN in depression}

SLN of curcumin and dexanabinol were prepared by He et al. to investigate the antidepressant effect in corticosterone induced depression model. SLN overcome the problems associated with curcumin like low solubility and low bioavailability with enhancement in antidepressant effect. The SLN showed an increase in curcumin level in the brain of mice and prolong the drug release which facilitates the antidepressant effect of curcumin ${ }^{55}$. Fatouh et al. prepared SLN of agomelatine; which is an antidepressant drug, to increase its bioavailability and target delivery to the brain. SLN were administered by intranasal route, which were found to be more effective than the intravenous route for brain targeting. The pharmacokinetic study proved that absolute bioavailability of agomelatine SLN was higher when compared to the oral suspension of valdoxan ${ }^{56}$.

\section{SLN in cosmetics}

Generally, UV radiations are extremely harmful to skin. SLN are useful carrier in cosmetics as they show controlled release of the drug, excellent blockage of UV rays, and uniform dispersion on skin without using any greasy oil ${ }^{57}$. Silymarin is a natural sunscreen agent having antioxidant properties. The SLN of silymarin was prepared and further formulated as sunscreen cream. After evaluation of cream, the sun protection factor in-vivo and in-vitro was 14.1 and 13.80 respectively and no changes appeared in accelerated stability studies, which showed that SLN of silymarin can be used for photoprotective action when formulated as sunscreen cream ${ }^{58}$.

\section{SLN for antimicrobial activity}

SLN can be used for the enhancement of antimicrobial activity of the natural products. In a study conducted by Fazly et al. the effectiveness of SLN against the human pathogen was investigated. For this, SLN of Eugenia caryophyllata essential oil was prepared and tested for antimicrobial activity against Pseudomonas aerug- 
inosa, Salmonella typhi, Staphylococcus aureus and Candida albicans. The study revealed that the SLN of Eugenia caryophyllata essential oil was either better or equivalent to the essential oil ${ }^{59}$. Jourghanian et al. conducted the antimicrobial studies to compare SLN of curcumin with the free curcumin via well diffusion test using E.coli, and S. aureus. The increased effect was observed with SLN than compare to free curcumin ${ }^{60}$.

\section{SLN for proteins and peptides delivery}

SLN have been proved as alternative carriers for the delivery of proteins, peptides, and antigens. They can entrap hydrophobic as well as hydrophilic proteins. SLN protects the peptides from proteolytic degradation and makes them able to cross the epithelial membrane and increase their bioavailability ${ }^{61}$ Xu et al. developed SLN of insulin containing an endoscopy escaping agent, HA2 peptide. During intracellular transport HA2 peptide SLN preserve the insulin and resulted in higher hypoglycemic effect in the diabetic rats ${ }^{62}$.

\section{SLN as a carrier for sustain drug delivery}

SLN can control the release of drug from its matrix. SLN used for the sustain delivery of hydrophilic and lipophilic drugs. In a study, conducted by Jourghanian et al., sustained release with improved antibacterial efficacy was obtained with curcumin loaded SLN ${ }^{60}$. Shazly et al. developed SLN of domperidone. The prepared SLN showed sustain release of domperidone up to $12 \mathrm{hrs}$ than compare to the conventional tablet, which showed immediate release. The results obtained from pharmacokinetic studies showed enhancement of oral bioavailability of domeperidone $^{63}$. Zhao et al. prepared SLN of yuxingcao essential oil (YEO) for pulmonary administration. The SLN showed prolonged release after intra-tracheal administration to rats. The study revealed that, SLN of YEO resulted in sustain effect after inhalation delivery than compare to YEO solution ${ }^{64}$. The SLN of Houttuynia cordata extract were prepared by Kim et al. for control drug delivery and high loading efficiency. The SLN of Houttuynia cordata extract showed sustain release of quercitrin from extract ${ }^{65}$.

\section{SLN for brain targeted drug delivery}

SLN can be targeted to the brain using the extremely small particle size of $50 \mathrm{~nm}$. SLN have the ability to cross the blood-brain barrier and used as a drug delivery system for the treatment of CNS diseases. The functionalization of SLN with ligands makes them appropriate for targeting the brain sites. A study conducted by Ramalingam et al. revealed that TMC (N-trimethyl chitosan) coated SLN improved brain delivery as compared to drug suspension. The surface modification with TMC protects them in the environment of the stomach, so that a large amount of drug can reach to systemic circulation and can cross the blood-brain barrier after oral administration ${ }^{12}$. Andrographolide is a natural product used in various brain disorders like cerebral ischemia. But the problem associated with its brain delivery is; low bioavailability, instability, and poor solubility. Graverini et al. developed SLN of andrographolide for brain delivery. In in-vivo studies, SLN were delivered to brain parenchyma outside the vascular bed; after crossing the blood-brain barrier $^{66}$. Loureiro et al. proved that SLN when combined with anti-transferrin receptor monoclonal antibody efficiently target the brain cells than compare to normal SLN. SLN-antibody conjugation was used for the delivery of grape extract with resveratrol to the brain to treat Alzhimer disease by inhibiting amyloid- $\beta$ peptide aggregation. The cellular uptake was found to be higher in SLN-antibody conjugation than the normal $\mathrm{SLN}^{67}$. The surface modification of SLN with apolipoprotein E-derived peptide (SLN-mApoE) was efficiently crossed the blood-brain barrier when tested using in-vitro model by Dal et al. The more confinement in the brain was found after pulmonary administration of SLN-mApoE, when compared to intravenous and intraperitoneal administration ${ }^{68}$. In a study, tocopherol succinate loaded SLN were developed for brain delivery of rivastigmine hydrogen tartrate for Alzheimer's disease. The prepared SLN were found with optimum particle size and high loading efficiency along with sustain release in-vitro ${ }^{69}$.

\section{Stealth nanoparticles}

SLN avoided by the immune system, so specific cells can also be targeted by SLN. Stealth SLN are efficiently used in malaria, lymphatic disease, and lung disease. Recent work on stealth solid lipid nanoparticles is shown in table 5 .

Table 5. Recent Work on Stealth Solid-lipid Nanoparticle

\begin{tabular}{|c|c|c|c|}
\hline Drug & Method & Inference & Reference \\
\hline Aloe-emodin (AE) & $\begin{array}{c}\text { Surface-functionalized } \\
\text { polyethylene glycol } \\
\text { liquid-crystalline } \\
\text { nanoparticles } \\
\text { (PEG-LCNPs) }\end{array}$ & $\begin{array}{c}\text { PEGylated LCNPs was found to be } \\
\text { promising nanocarrier for delivery } \\
\text { of Aloe-emodin to cancer cells. }\end{array}$ & 70 \\
\hline $\begin{array}{c}\text { Dimethyl fumarate, } \\
\text { Retinyl palmitate, } \\
\text { Progesterone, URB597 }\end{array}$ & $\begin{array}{c}\text { Modification of the } \\
\text { SLN surface with } \\
\text { polysorbate 80 }\end{array}$ & $\begin{array}{c}\text { The solubility of drugs was } \\
\text { improved by 4 to 8 fold. }\end{array}$ & 71 \\
\hline $\begin{array}{c}\text { Epigallocatechingallate } \\
\text { (EGCG) }\end{array}$ & $\begin{array}{c}\text { Encapsulation of } \\
\text { EGCG in solid lipid } \\
\text { nanoparticle }\end{array}$ & $\begin{array}{c}\text { Cytotoxicity of EGCG-SLN in MDA- } \\
\text { MB 231 human breast cancer and } \\
\text { DU-145 human prostate cancer cells } \\
\text { was found to be increased. }\end{array}$ & 72 \\
\hline $\begin{array}{c}\text { Lipoyl-memantine } \\
\text { (LA-MEM), }\end{array}$ & $\begin{array}{c}\text { Emuporation- solidifying } \\
\text { method }\end{array}$ & $\begin{array}{c}\text { LA-MEM loaded SLN were found to } \\
\text { be a potential candidate for in-vivo } \\
\text { studies in the brain. }\end{array}$ & 73 \\
\hline
\end{tabular}


In a study, stealth solid lipid nanoparticles (SSLN) were prepared by Wang et al. using polyoxyethylene stearate as stealth agent. The study of evading phagocytic uptake by mouse peritoneal macrophages using in- situ model concluded that, some amount of SLN and SSLN was uptake by macrophages. The interactions between cells and nanoparticles were perfectly evaluated using in situ model ${ }^{74}$.

\section{CONCLUSIONS}

Solid lipid nanoparticle system is a promising drug delivery system for the delivery of low aqueous soluble drugs as well as several natural products. The present review focused on various methods of preparation using various biodegradable polymers along with their applications in different fields. The lipids used in the preparation of lipid nanoparticles are included in the category of GRAS. SLN are considered as a potential and effective technique for controlled and targeted drug delivery of various drugs. Due to small particle size, SLN efficiently crosses bloodbrain barrier and hence can efficiently deliver the drug to the brain and used to treat various CNS related disorders. Further, due to its nature, SLN can be used for the delivery of genetic materials, proteins, peptides and various antimicrobial agents. The review also discussed the role of SLN as a carrier in cosmetic formulations. Moreover, with the numerous applications, SLN claims to overcome the shortcomings of other colloidal drug delivery system and further seems to be promising in designing of ligand-target drug delivery in various chronic diseases.

\section{CONFLICT OF INTERESTS}

The authors declare that there is no conflict of interest regarding the publication of this paper.

\section{REFERENCES}

1. Yun, Y. H.; Lee, B. K.; Park, K. Controlled Drug Delivery: Historical perspective for the next generation. $J$ Control Release. 2015, 219, 2-7.

2. Teja, V. R. C.; Chowdary, V. H.; Raju, Y. P.; Surendra, N.; Vardhan, R. V.; Reddy B. K. A Glimpse on solid lipid nanoparticles as drug delivery systems. Journal of Global Trends in Pharmaceutical Sciences. 2014, 5(2), 1649-57.

3. Lin, C. H.; Chen, C. H.; Lin, Z. C.; Fang, J. Y. Recent advances in oral delivery of drugs and bioactive natural products using solid lipid nanoparticles as the carriers. Journal of food and drug analysis. 2017, 25(2), 219-234.

4. Kumar, R.; Singh A.; Garg, N.; Siril, P. F. Solid lipid nanoparticles for the controlled delivery of poorly water soluble non-steroidal anti-inflammatory drugs. Ultrasonics Sonochemistry. 2018, 40, 686-696.

5. Behbahani, E. S.; Ghaedi, M.; Abbaspour, M.; Rostamizadeh, K. Optimization and characterization of ultrasound assisted preparation of Curcumin-loaded solid lipid nanoparticles: Application of central composite design, thermal analysis and X-ray diffraction techniques. Ultrasonics Sonochemistry. 2017, 38, 271-280.
6. Gupta, S.; Kesarla, R.; Chotai, N.; Misra, A.; Omri, A. Systematic Approach for the Formulation and Optimization of Solid Lipid Nanoparticles of Efavirenz by High Pressure Homogenization Using Design of Experiments for Brain Targeting and Enhanced Bioavailability. BioMed Research International. 2017, 2017.

7. Shafique, M.; Khan, M. A.; Khan, W. S.; Ahmad, W.; Khan, S. Fabrication, Characterization, and in vivo Evaluation of Famotidine Loaded Solid Lipid Nanoparticles for Boosting Oral Bioavailability. Journal of Nanomaterials. 2017, 2017.

8. Doktorovova, S.; Souto, E. B.; Silva, A. M. Hansen solubility parameters (HSP) for prescreening formulation of solid lipid nanoparticles (SLN): in vitro testing of curcumin-loaded SLN in MCF-7 and BT-474 cell lines. Pharmaceutical Development and Technology. 2018, 23(1), 96105.

9. Chakraborty, S.; Shukla, D.; Mishra, B.; Singh, S. Lipid - An emerging platform for oral delivery of drugs with poor bioavailability. European Journal of Pharmaceutics and Biopharmaceutics. 2009, 73, 1-15

10. Pandya, J. B.; Parmar, R. D.; Soniwala, M. M.; Chavda, J. R. Solid Lipid Nanoparticles: Overview on Excipients. Asian Journal of Pharmaceutical Technology \& Innovation. 2013, 1(3), 01-09.

11. Shah, R.; Eldridge, D.; Palombo, E.; Harding, I. Lipid nanoparticles: Production, characterization and stability. New York: Springer International Publishing. 2015, 11-22.

12. Ramalingam, P.; Ko, Y. T. Enhanced Oral Delivery of Curcumin from N-trimethyl Chitosan Surface-Modified Solid Lipid Nanoparticles: Pharmacokinetic and Brain Distribution Evaluations. Pharm Res. 2015, 32(2), 389-402.

13. Shi, L.; Li, Z.; Yu, L.; Jia, H.; Zheng, L. Effects of Surfactants and Lipids on the Preparation of Solid Lipid Nanoparticles Using Double Emulsion Method. Journal of Dispersion Science and Technology. 2011, 32(2), 254-259.

14. Jannin, V.; Blas, L.; Chevrier, S.; Miolane, C.; Demarne, F.; Spitzer, D. Evaluation of the digestibility of solid lipid nanoparticles of Glyceryl dibehenate produced by two techniques: Ultrasonication and spray-flash Evaporation. European Journal of Pharmaceutical Science. 2017, 111, 91-95.

15. Baek, J.; Na, Y., Cho, C. Sustained Cytotoxicity of Wogonin on Breast Cancer Cells by Encapsulation in Solid Lipid Nanoparticles. Nanomaterials. 2018, 8(3), 159.

16. Freitas, C.; Muller, R. H. Effect of light and temperature on zeta potential and physical stability in solid lipid nanoparticle $\left(\mathrm{SLN}^{\mathrm{TM}}\right)$ dispersions. International Journal of Pharmaceutics. 1998, 168, 221-9.

17. Ghada, A.; Fahmy, R. H. Diazepam-Loaded Solid Lipid Nanoparticles: Design and Characterization. American Association of Pharmaceutical Scientists. 2009, 10 (1), 211-219.

18. Rawat, M. K.; Jain, A.; Mishra, A.; Muthu, M. S.; Singh, S. Effect of lipid matrix on repaglinide loaded solid lipid nanoparticles for oral delivery. Therapeutic Delivery, 2010, 1, 63-73.

19. Tian, H.; Lu, Z.; Li, D.; Hu, J. Preparation and characterization of citral-loaded solid lipid nanoparticles. Food Chemistry. 2017, 248, 78-85.

20. Chantaburanan, T.; Teeranachaideekul, V.; Chantasart, D.; Jintapattanakit, A.; Junyaprasert, V. B.. Effect of binary solid lipid matrix of wax and triglyceride on lipid crystallinity, druglipid interaction and drug release of ibuprofen-loaded solid lipid nanoparticles (SLN) for dermal delivery. Journal of colloid and interface science. 2017, 504, 247-256.

21. Kheradmandnia, S.; Farahani, E. V.; Nosrati, M.; Atyabi, F. Preparation and characterization 
of ketoprofen-loaded solid lipid nanoparticles made from beeswax and carnauba wax. Nanomedicine: Nanotechnology, Biology, and Medicine. 2010, 6, 753-9.

22. Sun, J.; Bi, C.; Chan, H. M.; Sun, S.; Zhang, Q.; Zheng, Y. Curcumin-loaded solid lipid nanoparticles have prolonged in vitro antitumour activity, cellular uptake and improved in- vivo bioavailability. Colloids and Surfaces B: Biointerfaces. 2013, 111, 367-375.

23. Ganesan, P.; Ramalingam, P.; Karthivashan, G.; Ko, Y. T.; Choi, D. K. Recent developments in solid lipid nanoparticle and surface-modified solid lipid nanoparticle delivery systems for oral delivery of phyto-bioactive compounds in various chronic diseases. International Journal of $\mathrm{Na}$ nomedicine. 2018, 13, 1569-1583.

24. Baek, J. S.; Cho, C. W. Surface modification of solid lipid nanoparticles for oral delivery of curcumin: improvement of bioavailability through enhanced cellular uptake, and lymphatic uptake. European Journal of Pharmaceutics and Biopharmaceutics. 2017, 117, 132-140.

25. Wang, T.; Ma, X.; Lei, Y.; Luo, Y. Solid lipid nanoparticles coated with cross-linked polymeric double layer for oral delivery of curcumin. Colloids and Surfaces B: Biointerfaces. 2016, 148, $1-11$.

26. Khare, A.; Singh, I.; Pawar, P.; Grover, K. Design and evaluation of voriconazole loaded solid lipid nanoparticles for ophthalmic application. Journal of drug delivery. 2016, 2016.

27. Das, S.; Chaudhury, A. Recent Advances in Lipid Nanoparticle Formulations with Solid Matrix for Oral Drug Delivery. AAPS Pharm Sci Tech. 2011, 12(1), 62-76.

28. Chattopadhyay, P.; Shekunov, B. Y.; Yim, D.; Cipolla, D.; Boyd, B.; Farr, S. Production of solid lipid nanoparticle suspensions using supercritical fluid extraction of emulsions (SFEE) for pulmonary delivery using the AERx system. Advanced Drug Delivery Reviews. 2007, 59, 444453.

29. Wang, T.; Hu, Q.; Zhou, M.; Xia, Y.; Nieh, M.; Luo, Y. Development of "all natural" layer-bylayer redispersible solid lipid nanoparticles by nano spray drying technology. European Journal of Pharmaceutics and Biopharmaceutics. 2016, 107, 273-285.

30. Severino, P.; Silveira, E. F.; Loureiro, K.; Chaud, M. V.; Antonini, D.; Lancellotti, M.; Sarmento, V. H.; da Silva, C. F.; Santana, M. H. A.; Souto, E. B. Antimicrobial activity of polymyxinloaded solid lipid nanoparticles (PLX-SLN): Characterization of physicochemical properties and in vitro efficacy. European Journal of Pharmaceutical Sciences. 2017, 106, 177-184.

31. Ting, W.; Ning, W.; Yingying, Z.; Wancui, S.; Xingmei, G.; Tiefu, L. Solvent injection-lyophilization of tert-butyl alcohol/water cosolvent systems for the preparation of drug-loaded solid lipid nanoparticles. Colloids and Surfaces B: Biointerfaces. 2010, 79, 254-261.

32. Charcosset, C.; El-Harati, A.; Fessi, H. Preparation of solid lipid nanoparticles using a membrane contactor. Journal of Controlled Release. 2005, 108, 112-120.

33. Gaber, D. M.; Nafee, N.; Abdallah, O. Y. Myricetin solid lipid nanoparticles: Stability assurance from system preparation to site of action. European Journal of Pharmaceutical Sciences. 2017, 109, 569-580.

34. Daneshmand, S.; Golmohammadzadeh, S.; Jaafari, M. R.; Movaffagh, J.; Rezaee, M.; Sahebkar, A.; Malaekeh-Nikouei, B. Encapsulation challenges, the substantial issue in solid lipid nanoparticles characterization. Journal of cellular biochemistry. 2017. (Article in press)

35. Sinha, V. R.; Silki. Enhancement of In Vivo Efficacy and Oral Bioavailability of Aripiprazole with Solid Lipid Nanoparticles. AAPS PharmSciTech. 2018, 1-10. (Article in press)

36. Zhou, M.; Hou, J.; Zhong, Z.; Hao, N.; Lin, Y.; Li, C. Targeted delivery of hyaluronic acidcoated solid lipid nanoparticles for rheumatoid arthritis therapy. Drug Deliv. 2018, 25(1), 716-
722.

37. Daneshmand, S.; Jaafari, M. R.; Movaffagh, J.; Malaekeh-Nikouei, B.; Iranshahi, M.; Moghaddam, A. S.; Najaran, Z. T.; Golmohammadzadeh, S. Preparation, characterization, and optimization of auraptene-loaded solid lipid nanoparticles as a natural anti-inflammatory agent: In vivo and in vitro evaluations. Colloids and Surfaces B: Biointerfaces. 2018, 164, 332-339.

38. El-Housiny, S.; Shams Eldeen, M. A.; El-Attar, Y. A.; Salem, H. A.; Attia, D.; Bendas, E. R.; El-Nabarawi, M. A. Fluconazole-loaded solid lipid nanoparticles topical gel for treatment of pityriasis versicolor: formulation and clinical study. Drug delivery. 2018, 25(1), 78-90.

39. Ud Din, F.; Mustapha, O.; Kim, D. W.; Rashid, R.; Park, J. H.; Choi, J. Y.; Ku, S. K.; Yong, C. S.; Kim, J. O.; Choi, H. G. Novel dual-reverse thermosensitive solid lipid nanoparticle-loaded hydrogel for rectal administration of flurbiprofen with improved bioavailability and reduced initial burst effect. European Journal of Pharmaceutics and Biopharmaceutics. 2015, 94, 64-72.

40. Sánchez-López, E.; Espina, M.; Doktorovova, S.; Souto, E. B.; García, M. L. Lipid nanoparticles (SLN, NLC): overcoming the anatomical and physiological barriers of the eye-part I-barriers and determining factors in ocular delivery. European Journal of Pharmaceutics and Biopharmaceutics. 2017, 110, 70-75.

41. Haque, S.; Whittaker, M.; McIntosh, M. P.; Pouton, C. W.; Phipps, S.; Kaminskas, L. M. A comparison of the lung clearance kinetics of Solid lipid nanoparticles and Liposomes by following the $3 \mathrm{H}$-labelled structural lipids after pulmonary delivery in rats. European Journal of Pharmaceutics and Biopharmaceutics. 2018, 125, 1-12.

42. Rosiere, R.; Van Woensel, M.; Gelbcke, M.; Mathieu, V.; Hecq, J.; Mathivet, T.; Vermeersch, M.; Van Antwerpen, P. G.; Amighi, K.; Wauthoz, N. A new folate-grafted chitosan derivative to improve the delivery of paclitaxel-loaded solid lipid nanoparticles for lung tumour therapy by inhalation. Molecular pharmaceutics. 2018, 15(3), 899-910.

43. Botto, C.; Mauro, N.; Amore, E.; Martorana, E.; Giammona, G.; Bondì, M. L. Surfactant effect on the physicochemical characteristics of cationic solid lipid nanoparticles. International journal of pharmaceutics. 2017, 516(1-2), 334-341.

44. Kotmakçi, M.; Çetintaş, V. B.; Kantarci, A. G. Preparation and characterization of lipid nanoparticle/pDNA complexes for STAT3 downregulation and overcoming chemotherapy resistance in lung cancer cells. International journal of pharmaceutics. 2017, 525(1), 101-111.

45. Sharma, G.; Chopra, K.; Puri, S.; Bishnoi, M.; Rishi, P.; Kaur, I. P. Topical delivery of TRPsiRNA-loaded solid lipid nanoparticles confer reduced pain sensation via TRPV1 silencing, in rats. Journal of drug targeting. 2018, 26(2), 135-149.

46. Akbaba, H., Akbaba, G.E. and Kantarci, A.G. Development and evaluation of antisense shRNA-encoding plasmid loaded solid lipid nanoparticles against 5-a reductase activity. Journal of Drug Delivery Science and Technology. 2018, 44, 270-277.

47. Costa, A.; Sarmento, B.; Seabra, V. Mannose-functionalized solid lipid nanoparticles are effective in targeting alveolar macrophages. European Journal of Pharmaceutical Sciences. 2018, 114, 103-113.

48. Maretti, E.; Costantino, L.; Rustichelli, C.; Leo, E.; Croce, M. A.; Buttini, F.; Truzzi, E.; Iannuccelli, V. Surface engineering of Solid Lipid Nanoparticle assemblies by methyl $\alpha-\alpha-$ mannopyranoside for the active targeting to macrophages in anti-tuberculosis inhalation therapy. International journal of pharmaceutics. 2017, 528(1-2), 440-451.

49. Vieira, A. C.; Chaves, L. L.; Pinheiro, S.; Pinto, S.; Pinheiro, M.; Lima, S. C.; Ferreira, D.; Sarmento, B.; Reis, S. Mucoadhesive chitosan-coated solid lipid nanoparticles for better management of tuberculosis. International journal of pharmaceutics. 2018, 536(1), 478-485. 
50. Tang, J.; Ji, H.; Ren, J.; Li, M.; Zheng, N.; Wu, L. Solid lipid nanoparticles with TPGS and Brij 78: A co-delivery vehicle of curcumin and piperine for reversing P-glycoprotein-mediated multidrug resistance in vitro. Oncology Letters. 2017, 13, 389-395.

51. Chirio, D.; Gallarate, M.; Peira, E.; Battaglia, L.; Serpe, L.; Trotta, M. Formulation of curcumin-loaded solid lipid nanoparticles produced by fatty acids coacervation technique. Journal of Microencapsulation. 2011, 28(6), 537-548.

52. Chuang, C. H.; Wu, P. C.; Tsai, T. H.; Fang, Y. P.; Tsai, Y. H.; Cheng, T. C.; Huang, C. C.; Huang, M. Y.; Chen, F. M.; Hsieh, Y. C.; Lin, W. W. Development of pH-Sensitive Cationic PEGylated Solid Lipid Nanoparticles for Selective Cancer-Targeted Therapy. Journal of Biomedical Nanotechnology. 2017, 13(2), 192-203.

53. Badawi, N. M.; Teaima, M. H.; El-Say, K. M.; Attia, D. A.; El-Nabarawi, M. A.; Elmazar, M. M. Pomegranate extract-loaded solid lipid nanoparticles: design, optimization, and in vitro cytotoxicity study. Int $J$ Nanomedicine. 2018, 13, 1313-1326.

54. Oliveira, M. S.; Lima, B. H. S.; Goulart, G. A. C.; Mussi, S. V.; Borges, G. S. M.; Oréfice, R. L.; Ferreira, L. A. M. Improved Cytotoxic Effect of Doxorubicin by Its Combination with Sclareol in Solid Lipid Nanoparticle Suspension. Journal of nanoscience and nanotechnology. 2018, 18(8), 5609-5616.

55. He, X.; Yang, L.; Wang, M.; Zhuang, X.; Huang, R.; Zhu, R.; Wang, S. Targeting the Endocannabinoid/CB1 Receptor System for Treating Major Depression Through Antidepressant Activities of Curcumin and Dexanabinol-Loaded Solid Lipid Nanoparticles. Cellular Physiology and Biochemistry. 2017, 42, 2281-94.

56. Fatouh, A. M.; Elshafeey, A. H.; Abdelbary, A. Intranasal agomelatine solid lipid nanoparticles to enhance brain delivery: formulation, optimization and in vivo pharmacokinetics. Drug design, development and therapy. 2017, 11, 1815-1825.

57. Jose, J.; Netto, G. Role of solid lipid nanoparticles as photoprotective agents in cosmetics. Journal of Cosmetic Dermatology. 2018. (article in press)

58. Netto MPharm, G.; Jose, J. Development, characterization, and evaluation of sunscreen cream containing solid lipid nanoparticles of silymarin. Journal of cosmetic dermatology. $\mathbf{2 0 1 7}$ (Article in press)

59. Fazly, B. B. S.; Khameneh, B.; Namazi. N.; Iranshahi, M.; Davoodi, D.; Golmohammadzadeh, S. Solid Lipid Nanoparticles (SLN) carrying Eugenia caryophyllata essential oil: The novel nanoparticulate systems with broad-spectrum antimicrobial activity. Lett Appl Microbiol. 2018, article in press.

6o. Jourghanian, P.; Ghaffari, S.; Ardjmand, M.; Haghighat, S.; Mohammadnejad, M. Sustained release Curcumin loaded Solid Lipid Nanoparticles. Adv Pharm Bull. 2016, 6(1), 17-2.

61. Dumont, C.; Bourgeois, S.; Fessi, H.; Jannin, V. Lipid-based nanosuspensions for oral delivery of peptides, a critical review. International journal of pharmaceutics. 2018, 541(1-2), 117-135.

62. Xu, Y.; Zheng, Y.; Wu, L.; Zhu, X.; Zhang, Z.; Huang, Y. Novel Solid Lipid Nanoparticle with Endosomal Escape Function for Oral Delivery of Insulin. ACS applied materials \& interfaces. 2018, 10(11), 9315-9324

63. Shazly, G. A.; Alshehri, S.; Ibrahim, M. A.; Tawfeek, H. M.; Razik, J. A.; Hassan, Y. A.; Shakeel, F. Development of Domperidone Solid Lipid Nanoparticles: In Vitro and In Vivo Characterization. AAPS PharmSciTech. 2018, Article in press.

64. Zhao, Y.; Chang, Y. X.; Hu, X.; Liu, C. Y.; Quan, L. H.; Liao, Y. H. Solid lipid nanoparticles for sustained pulmonary delivery of Yuxingcao essential oil: preparation, characterization and in vivo evaluation. International journal of pharmaceutics. 2017, 516(1-2), 364-371.

65. Kim, J. H.; Baek, J. S.; Park, J. K.; Lee, B. J.; Kim, M. S.; Hwang, S. J.; Lee, J. Y.; Cho, C. W. Development of Houttuynia cordata Extract-Loaded Solid Lipid Nanoparticles for Oral Delivery High Drug Loading Efficiency and Controlled Release. Molecules. 2017, 22(12), 2215.

66. Graverini, G.; Piazzini, V.; Landucci, E.; Pantano, D.; Nardiello, P.; Casamenti, F.; PellegriniGiampietro, D. E.; Bilia, A. R.; Bergonzi, M. C. Solid lipid nanoparticles for delivery of andrographolide across the blood-brain barrier: in vitro and in vivo evaluation. Colloids Surf B Bioint erfaces. 2018, 161, 302-313.

67. Loureiro, J. A.; Andrade, S.; Duarte, A.; Neves, A. R.; Queiroz, J. F.; Nunes, C.; Sevin, E Fenart, L.; Gosselet, F.; Coelho, M. A.; Pereira, M. C. Resveratrol and grape extract-loaded solid lipid nanoparticles for the treatment of Alzheimer's disease. Molecules. 2017, 22(2), 277.

68. Dal Magro, R.; Ornaghi, F.; Cambianica, I.; Beretta, S.; Re, F.; Musicanti, C.; Rigolio, R.; Donzelli, E.; Canta, A.; Ballarini, E.; Cavaletti, G. ApoE-modified solid lipid nanoparticles: A feasible strategy to cross the blood-brain barrier. Journal of Controlled Release. 2017, 249, 103 110.

69. Malekpour-Galogahi, F.; Hatamian-Zarmi, A.; Ganji, F.; Ebrahimi-Hosseinzadeh, B.; Nojoki F.; Sahraeian, R.; Mokhtari-Hosseini, Z. B. Preparation and optimization of rivastigmine-loaded tocopherol succinate-based solid lipid nanoparticles. Journal of liposome research. 2017, 1-10.

70. Freag, M. S.; Elnaggar, Y. S.; Abdelmonsif, D. A.; Abdallah, O. Y. Stealth, biocompatible monoolein-based lyotropic liquid crystalline nanoparticles for enhanced aloe-emodin delivery to breast cancer cells: in vitro and in vivo studies. International journal of nanomedicine. $\mathbf{2 0 1 6}$ 11,4799

71. Esposito, E.; Drechsler, M.; Mariani, P.; Carducci, F.; Servadio, M.; Melancia, F.; Ratano, P.; Campolongo, P.; Trezza, V.; Cortesi, R.; Nastruzzi, C. Lipid nanoparticles for administration of poorly water soluble neuroactive drugs. Biomedical microdevices. 2017, 19(3), 44

72. Radhakrishnan, R.; Kulhari, H.; Pooja, D.; Gudem, S.; Bhargava, S.; Shukla, R.; Sistla, R. Encapsulation of biophenolic phytochemical EGCG within lipid nanoparticles enhances its stability and cytotoxicity against cancer. Chemistry and physics of lipids. 2016, 198, 51-60.

73. Laserra, S.; Basit, A.; Sozio, P.; Marinelli, L.; Fornasari, E.; Cacciatore, I.; Ciulla, M.; Türkez, H.; Geyikoglu, F.; Di Stefano, A. Solid lipid nanoparticles loaded with lipoyl-memantine codrug: preparation and characterization. International journal of pharmaceutics. 2015, 485(1-2), 183191.

74. Wang, Y.; Wu, W. In situ evading of phagocytic uptake of stealth solid lipid nanoparticles by mouse peritoneal macrophages. Drug Deliv. 2006, 13, 189-92. 